\title{
On the Moduli Space of Anti-Self-Dual Yang-Mills Connections on Kähler Surfaces
}

By

\author{
Mitsuhiro IToH*
}

\section{$\S 0$. Introduction and Main Theorems}

The purpose of this paper is to give a complete proof of the result announced in [7]. In fact in this paper we discuss the dimension of moduli space consisting of anti-self-dual solutions of Yang-Mills equation in the case where the base space is Kähler and we obtain the dimension formula which is similar to the case of moduli space of self-dual solutions over a self-dual base space, given by Atiyah, Hitchin and Singer [2]. Further we get on a compact Kähler surface a suggestive observation that the moduli space of anti-self-dual Yang-Mills connections may have a close relation to moduli space of holomorphic vector bundles.

Yang-Mills connections, namely, solutions to Yang-Mills equation have originated from field theory in physics $([12])$. Yang-Mills equation is considered as a generalization of Maxwell equation from a viewpoint of non-abelian gauge group. Mathematically, Yang-Mills connections are formulated by the aid of notions of connections on a principal fibre bundle.

Let $P$ be a principal bundle over a compact oriented Riemannian 4-manifold $M$ with a compact semi-simple Lie group $G$. Let $E$ be an associated complex vector bundle. A functional $\mathscr{Y} \mathscr{M}$ is defined over the space $\mathcal{C}_{E, G}$ consisting of all $G$-connections on $E$ by $\mathscr{G} \mathscr{M}(\nabla)=(1 / 2)\left\|R^{\nabla}\right\|^{2}$ for the curvature form $R^{\nabla}$ of $\nabla$. The Euler-Lagrangian equation of the functional is written by $d^{\nabla}\left(* R^{\nabla}\right)=0$. A $G$-connection which gives a solution to this equation is called a Yang-Mills $G$ connection. From Bianchi's identity an (anti-)self-dual $G$-connection which satisfies that $* R^{\nabla}= \pm R^{\nabla}$ is a special Yang-Mills $G$-connection. If we denote by $\mathcal{A}_{E, G}^{ \pm}$the set of all (anti-)self-dual $G$-connections, then the space $\mathscr{M}_{E, G}^{ \pm}$, which is a quotient space of $A_{E, G}^{ \pm}$, modulo the group of gauge transformations $\mathcal{G}_{P}$ represents moduli space of essentially distinct (anti-)self-dual $G$-connections.

Now suppose that $M$ is a Kähler surface. Then $M$ admits the canonical orientation induced from the complex structure of $M$. With respect to the space of infinitesimal deformations of anti-self-dual $G$-connections, that is, the tangent

Communicated by M. Sato, June 1, 1981.

* Institute of Mathematics, University of Tsukuba, Ibaraki 305, Japan. 
space of the moduli space $\mathscr{M}_{\bar{E}, G}$, we have the following theorem.

Theorem 0.1. Let $M$ be a compact Kähler surface of positive scalar curvature and $P$ a smooth principal bundle with a compact semi-simple Lie group $G$. Let $E$ be a complex vector bundle associated with $P$. Then the space of infinitesimal deformations of irreducible anti-self-dual G-connections has the dimension

$$
- \text { Pont }_{1}\left(\mathrm{~g}_{P}^{C}\right)-\frac{1}{2} \operatorname{dim} G(\chi+\tau) \text {. }
$$

Here $\mathrm{g}_{P}$ is the Lie algebra bundle associated with $P$ and $\mathrm{g}_{P}^{C}$ is its complexification, and $\operatorname{Pont}_{1}(\cdot), \chi$ and $\tau$ are the first Pontrjagin number, the Euler number of $M$ and the signature of $M$, respectively.

This theorem is shown by the aid of propositions (Proposition 2.4, Theorem 3.1 and Theorem 4.1) mostly parallel to the proof of Theorem 6.1 in [2]. However, the fact that an anti-self-dual 2 -form is characterized as a form of type $(1,1)$, orthogonal to the fundamental form $\Omega$ plays an essential role in proving our theorem (Lemma 2.1). If we are given an irreducible anti-self-dual $G$-connection $\nabla$, then we observe in Proposition 2.4 that the space of infinitesimal deformations of $\nabla$ preserving anti-self-duality is exactly the first cohomology group $H^{1}$ of a certain elliptic complex. That the 0 -th cohomology group $H^{0}$ vanishes follows immediately from the irreducibility of $\nabla$. Further under the assumption that $M$ has positive scalar curvature the vanishing theorem of the second cohomology group $H^{2}$ is shown by a Bochner type estimate of the Laplacians associated with the complex in Theorem 3.1. Then the dimension of the space of infinitesimal deformations is obtained by applying the Atiyah-Singer index theorem to the complex in the similar manner to the Riemann-Roch-Hirzebruch theorem for a holomorphic vector bundle (Theorem 4.1).

If we apply Kuranishi's method to Theorem 0.1 in the same manner as in the proof of Theorem 6.1 in [2], then we obtain the following.

Theorem 0.2. Under the same assumption as that in Theorem 0.1, the moduli space $\mathscr{M}_{\bar{E}, G}$ of irreducible anti-self-dual G-connections has a structure of manifold of dimension

$$
- \text { Pont }_{1}\left(g_{P}^{C}\right)-\frac{1}{2} \operatorname{dim} G(\chi+\tau),
$$

if it is not empty.

Remark 1. Theorem 6.1 in [2] is stated as follows; the moduli space $\mathscr{M}_{E, G}^{+}$ of irreducible self-dual $G$-connections over a compact self-dual Riemannian 4manifold of positive scalar curvature is either empty or a manifold of dimension

$$
\text { Pont }_{1}\left(g_{P}^{C}\right)-\frac{1}{2} \operatorname{dim} G(\chi-\tau) \text {. }
$$

However in our situation $\operatorname{dim} \mathscr{M}_{E, G}^{+}$over a Kähler surface $M$ with the canonical 
orientation does not necessarily coincide with the above formula, because the second cohomology group $H^{2}$ may not vanish, even if $M$ is of positive scalar curvature.

Remark 2. An oriented Riemannian 4-manifold is called to be (anti-)selfdual if its Weyl's conformal curvature tensor, regarded as an End $(T M)$-valued form, is (anti-)self-dual ([2]). From the result of [6] together with Corollary 14.5 in [11], a compact Kähler surface is self-dual if and only if it is a complex space form or a ruled surface (i.e., a $P_{1}(\boldsymbol{C})$-bundle over a compact complex curve) covered by the product of $P_{1}(C)$ with positive constant curvature $c$ and the unit disk $D^{1}$ with constant curvature $-c$. Therefore, a compact self-dual Kähler surface of positive scalar curvature is $P_{2}(C)$ of standard metric. The product $P_{1}(\boldsymbol{C}) \times P_{1}(\boldsymbol{C})$ and a ruled surface $F=\left\{\left(z_{0}: z_{1}: z_{2}\right) \times(s, t) \in P_{2}(\boldsymbol{C}) \times P_{1}(\boldsymbol{C})\right.$; $\left.s z_{0}-t z_{1}=0\right\}$ with standard metrics are examples of simply connected compact Kähler surfaces of positive scalar curvature, both of which are not self-dual. It is noticed that any ruled surface admits a Kähler metric of positive scalar curvature ([13]). Our theorem can be applied to these Kähler surfaces.

Suppose that a complex vector bundle $E$ associated with a principal bundle $P$ over a compact Kähler surface admits an anti-self-dual $G$-connection $\nabla$. Then from Note after Proposition 2.2 in Section 2, $E$ admits a hermitian structure $h$ and a holomorphic structure $J$ such that $\nabla h=0$ and $\nabla J=0$. Since the curvature form $R^{\nabla}=\left(R_{j}^{\nabla i}\right)$ is $\mathfrak{g}$-valued 2 -form with respect to a $G$-frame $(\mathfrak{g} \subseteq \mathfrak{g} \mathfrak{u}(n), n=\operatorname{rank} E)$, $c_{1}(E)$ is represented by $(1 /(2 \pi \sqrt{-1})) \sum_{i=1}^{n} R_{i}^{\nabla i}=0$. Then there are topological restrictions on $E, c_{1}(E)=0$ and $c_{2}(E)=(1 / 2)\left(c_{1}^{2}(E)-\operatorname{Pont}_{1}(E)\right)>0$, if $\nabla$ is irreducible.

Proposition 0.3. Let $M$ be a compact Kähler surface and $E$ be a complex vector bundle associated with a principal bundle $P$ over $M$ with $G$. If there exists an irreducible anti-self-dual $G$-connection $\nabla$ on $E$, then $H^{\circ}(M ; \mathcal{O}(E))=0$ with respect to the holomorphic structure $J$. And if further $E$ is a bundle of rank 2, defined over $P_{2}(\boldsymbol{C})$, then $E$ is stable as a holomorphic vector bundle.

This proposition is verified as follows. Suppose that the holomorphic vector bundle $E$ with the holomorphic structure $J$ has a nontrivial global holomorphic section $\phi$. Since $\sum g^{\mu \bar{\nu}} R_{\mu \bar{\nu}}{ }^{i}=0$ (Proposition 2.2), $\phi$ is parallel with respect to $\nabla$ ([8]) and hence it vanishes nowhere. Then the holonomy group is reduced to a proper closed subgroup of $G$. This is a contradiction to the irreducibility of $\nabla$. Hence we have that $H^{0}(M ; \mathcal{O}(E))=0$. The last part of this proposition is shown by the aid of Lemma 1.2.5, page 165 in [10]. For the definition of a stable vector bundle, refer to [10].

Remark. The moduli space $\mathscr{M}_{\bar{E}, G}$ of irreducible anti-self-dual $S U(2)$-connections has dimension $2(4 n-3)$ for a complex vector bundle $E$ of rank 2, defined over $P_{2}(C)$, where $c_{1}(E)=0$ and $c_{2}(E)=n$. This dimension corresponds to the 
fact that the moduli space of holomorphic vector bundles of rank 2 with $c_{1}=0$, $c_{2}=n$ over $P_{2}(\boldsymbol{C})$ is a variety of complex dimension $4 n-3$ ([4]).

In Section 1 we define a $G$-connection and its curvature form on a vector bundle and treat notions of Yang-Mills $G$-connections and (anti-)self-dual $G$ connections. This section also provides a note that is elemental when we covariantly exterior differentiate on a complex manifold.

In Section 2 we give a characterization of anti-self-dual $G$-connections over a Kähler surface and present a proposition which states a relation between infinitesimal deformations of anti-self-dual $G$-connections and the first cohomology group of a certain elliptic complex.

The vanishing theorems of the 0 -th and the second cohomology groups of the complex are obtained in Section 3 and the index of the complex is calculated in Section 4.

In the remainder of this paper we devote ourselves to verification of Bochner's type formulas (Lemmas 3.2 and 3.3) that are key formulas in proving the vanishing theorem of the second cohomology group in Section 3.

For basic references about Yang-Mills $G$-connections, see [2] and [5], and for fundamental notions of Kähler manifolds, refer to [9] and [14].

The author is very grateful to Dr. Mulase for helpful conversations, especially for an advice on the stability condition of vector bundles.

\section{$\S 1$. Connections and Curvature Forms on a Vector Bundle}

Let $M$ be a compact oriented Riemannian manifold and $P$ a smooth principal bundle over $M$ with a compact semi-simple Lie group $G$. Then the Lie group bundle $G_{P}$ and the Lie algebra bundle $\mathrm{g}_{P}$ are naturally induced from $P$ by $G_{P}=$ $P \times{ }_{c} G$ and $g_{P}=P \times_{A d} g$ respectively, where $c: G \rightarrow A u t G$ is the group conjugation and $A d$ is the adjoint representation of $G$ to the Lie algebra $g$ of $G$. A global smooth section of $G_{P}$ is called a gauge transformation of $P$ and $\Gamma\left(M ; G_{P}\right)$, which is denoted by $\mathcal{G}_{P}$, is called the group of gauge transformations of $P$.

A locally faithful representation $\rho$ of $G$ to $G L(n ; C)$ induces a smooth complex vector bundle $E=P \times{ }_{\rho} C^{n}$ over $M$. Every $s$ of $P$ over $x$ of $M$ naturally defines a linear isomorphism of $C^{n}$ onto the fibre $E_{x}$ by $v \rightarrow s \cdot v$. Fix a frame $\left\{e_{i}\right\}_{1 \leqq i \leqq n}$ of $\boldsymbol{C}^{n}$. Then a local smooth section $s$ of $P$ over an open $U$ of $M$ induces a local frame $\left\{t_{i}\right\}_{1 \leqq i \leqq n}$ of $E$ over $U$ given by $t_{i}=s \cdot e_{i}, 1 \leqq i \leqq n$. The frame $\left\{t_{i}\right\}_{1 \leqq i \leqq n}$ is called a $G$-frame.

We denote by $A^{p}(E)$ the set of all smooth $E$-valued $p$-forms $\Gamma\left(M ; \Lambda^{p} \otimes E\right)$ for $p \geqq 0$.

A connection $\nabla$ on $E$ is a linear differential operator of first order from $A^{0}(E)$ to $A^{1}(E)$ which satisfies that $\nabla(f \phi)=d f \otimes \phi+f \nabla \phi$ for $f \in C^{\infty}(M)$ and $\phi \in$ $A^{0}(E)$.

Since $\nabla t_{i}$ is represented by $\nabla t_{i}=\sum_{j} A_{i}^{\nabla j} t_{j}$ for a matrix valued 1-form $A^{\nabla}=$ 
$\left(A_{\imath}^{7_{\imath}}\right)$, the covariant derivative of $\phi=\sum_{i} \phi^{i} t_{i}$ is written by

$$
\nabla \phi=\sum_{i}\left(d \phi^{i}+\sum_{j} \phi^{j} A_{j}^{\nabla i}\right) t_{i} .
$$

The matrix valued 1-form $A^{\nabla}=\left(A_{i}^{\nabla j}\right)=\left(\Sigma_{\mu} A^{\nabla}{ }_{\mu 2}^{j} d x^{\mu}\right)$ is called the connection form of $\nabla$ with respect to $\left\{t_{i}\right\}$.

Definition 1.1. A connection $\nabla$ on $E$ is called a $G$-connection if its connection form $A^{\nabla}$ takes values in $\mathrm{g}$ with respect to any $G$-frame, where $\mathrm{g}$ is identified with a subalgebra of $\mathrm{gl}(n ; C)$ through $\rho$.

We denote by $\mathcal{C}_{E, G}$ the set of all $G$-connections on $E$. Since the difference of $G$-connections represents a $\mathrm{g}_{P}$-valued 1 -form, $\mathcal{C}_{E, G}$ has an affine structure, that is, $\mathcal{C}_{E, G}=\nabla^{0}+A^{1}\left(\mathfrak{g}_{P}\right)$ for a fixed $\nabla^{0}$ of $\mathcal{C}_{E, G}$.

We notice that there is a one-to-one correspondence between $\mathcal{C}_{E, G}$ and the set of all connections in $P$ in the following manner. A connection in $P$ with connection form $\omega$ defines a $G$-connection $\nabla^{\omega}$ on $E$ by $\nabla^{\omega} t_{i}=\Sigma_{j}\left(\rho s^{*} \omega\right)_{2}^{j} t_{j}, 1 \leqq i \leqq n$, for a local section $s$ of $P$ and a $G$-frame $\left\{t_{\imath}\right\}$ defined by $s$ ([2]). Conversely, for any $\nabla \in \mathcal{C}_{E, G}$ we can define a connection in $P$ with connection form $\omega$ such that $\nabla$ coincides with $\nabla^{\omega}$.

We introduce the notion of the irreducibility of a $G$-connection. A $G$-connection is called irreducible if its holonomy group is an open subgroup of $G$ ([2]). The set of irreducible $G$-connections is an open subset of $\mathcal{C}_{E, G}$ in a certain topology.

Given a $G$-connection $\nabla$ we can define a differential operator of first order $d^{\nabla}: A^{p}(E) \rightarrow A^{p+1}(E)$, called the covariant exterior differentiation as follows:

$$
d^{\nabla}\left(\Sigma_{i} \theta^{i} \otimes t_{i}\right)=\sum_{i}\left(d \theta^{i}+(-1)^{p} \sum_{\jmath} \theta^{j} \wedge A^{\nabla i}\right) t_{i}
$$

for local $p$-forms $\theta^{i}, 1 \leqq i \leqq n$.

An $\operatorname{End}(E)$-valued 2-form $R^{\nabla}=\left(R_{j}^{\nabla \imath}\right)$ given by

$$
R_{j}^{\nabla i}=d A_{j}^{\nabla i}-\Sigma_{h} A_{j}^{\nabla h} \wedge A_{h}^{\nabla \imath}
$$

is called the curvature form of $\nabla$. Then we have

$$
d^{\nabla} \circ \nabla(\phi)=\sum_{i, j} \phi^{j} R^{\nabla i} t_{i}
$$

for $\phi \in A^{0}(E)$. The similar formula also holds for $\theta$ of $A^{p}(E), p \geqq 1$;

$$
d^{\nabla} \cdot d^{\nabla}(\theta)=\sum_{i, j} \theta^{j} \wedge R^{\nabla i} t_{i}
$$

Since $R^{\nabla}$ takes values in $\mathrm{g}$ locally and is transformed in the form of the adjoint representation of $G$ with respect to a transformation of $G$-frames, $R^{\nabla}$ is regarded as a $\mathfrak{g}_{P}$-valued 2 -form, that is, $R^{\nabla} \in A^{2}\left(\mathfrak{g}_{P}\right)$.

The bundle $\mathrm{g}_{P}$ is identified with a subbundle of $\operatorname{End}(E)$ through $\rho$. We introduce a connection on $g_{P}$ by the aid of $\nabla$ in the following natural manner;

$$
(\nabla \Phi)(\phi)=\nabla(\Phi(\phi))-\Phi(\nabla \phi)
$$

for $\Phi \in A^{0}\left(\mathfrak{g}_{P}\right)$ and $\phi \in A^{0}(E)$. Then for $\Phi=\sum_{i, j} \Phi_{j}^{i} t_{\imath} \otimes t^{j} \in A^{0}\left(\mathfrak{g}_{P}\right)$ ( $t^{j}$ is the dual of 
$\left.t_{j}\right) \nabla \Phi$ is written locally by

$$
(\nabla \Phi)_{j}^{i}=d\left(\Phi_{\jmath}^{i}\right)+\left[\Phi, A^{\nabla}\right]_{j}^{i} .
$$

Introduce a bilinear mapping $[\cdot \wedge \cdot]: A^{p}\left(\mathrm{~g}_{P}\right) \times A^{q}\left(\mathrm{~g}_{P}\right) \rightarrow A^{p+q}\left(\mathrm{~g}_{P}\right)$ by

$$
[\Phi \wedge \Psi]_{j}^{i}=\Sigma_{h}\left\{\Phi_{\jmath}^{h} \wedge \Psi_{h}^{i}-(-1)^{p q} \Psi_{j}^{h} \wedge \Phi_{h}^{i}\right\}
$$

If we set $p=q=0$, then $[\Phi \wedge \Psi]$ represents the ordinary bracket product $[\Phi, \Psi]$ of matrices $\Phi$ and $\Psi$. Then the covariant exterior differentiation $d^{\nabla}: A^{p}\left(\mathrm{~g}_{P}\right) \rightarrow$ $A^{p+1}\left(\mathrm{~g}_{P}\right)$ has the following expression;

$$
\left(d^{\nabla} \Phi\right)_{\jmath}^{i}=d\left(\Phi_{\jmath}^{i}\right)+(-1)^{p}\left[\Phi \wedge A^{\nabla}\right]_{\jmath}^{i}
$$

for $\Phi \in A^{p}\left(\mathrm{~g}_{P}\right)$.

We have the following identities;

$$
d^{\nabla} R^{\nabla}=0 \quad \text { (Bianchi's identity) }
$$

and

$$
d^{\nabla} \circ d^{\nabla}(\Phi)=\left[\Phi \wedge R^{\nabla}\right], \quad \Phi \in A^{p}\left(\mathfrak{g}_{P}\right) .
$$

These are easily verified by the aid of the formula $R^{\nabla}=d A^{\nabla}-(1 / 2)\left[A^{\nabla} \wedge A^{\nabla}\right]$ together with the following properties of $[\cdot \wedge \cdot]$;

$$
\begin{gathered}
{[\Phi \wedge \Psi]=-(-1)^{p q}[\Psi \wedge \Phi],} \\
d[\Phi \wedge \Psi]=[d \Phi \wedge \Psi]+(-1)^{p}[\Phi \wedge d \Psi]
\end{gathered}
$$

and

$$
[[\Phi \wedge \Psi] \wedge \Theta]+(-1)^{p q+q r}[[\Psi \wedge \Theta] \wedge \Phi]+(-1)^{q r+p r}[[\Theta \wedge \Phi] \wedge \Psi]=0
$$

for a local $\mathrm{g}$-valued $p$-form $\Phi$, a local $\mathrm{g}$-valued $q$-form $\Psi$ and a local $\mathrm{g}$-valued $r$-form $\Theta$.

Let $\Phi$ be a $\mathfrak{g}_{P^{-}}$-valued $p$-form. Then we have the following two local expressions of $\Phi$;

$$
\Phi=\frac{1}{p !} \sum_{\mu_{1}, \mu_{2}, \cdots, \mu_{p}} \Phi_{\mu_{1} \mu_{2} \cdots \mu_{p}} d x^{\mu_{1}} \wedge d x^{\mu_{2}} \wedge \cdots \wedge d x^{\mu_{p}}
$$

and

$$
\Phi=\frac{1}{p !} \sum_{i, j} \sum_{\mu_{1}, \mu_{2}, \cdots, \mu_{p}} \Phi_{\mu_{1} \mu_{2} \cdots \mu_{p}{ }^{j}} d x^{\mu_{1}} \wedge d x^{\mu_{2}} \wedge \cdots \wedge d x^{\mu_{p}} \otimes t_{i} \otimes t^{j}
$$

for g-valued local functions $\Phi_{\mu_{1} \cdots \mu_{p}}$ and local functions $\Phi_{\mu_{1} \cdots \mu_{p}}$, which are skew symmetric with respect to $\mu_{1}, \cdots, \mu_{p}$. In the following we use a suitable expression as occasion requires.

For a $G$-connection $\nabla$ we define a linear differential operator from $\Gamma\left(M ; \stackrel{p}{\otimes} T^{*} M \otimes \mathrm{g}_{P}\right)$ to $\Gamma\left(M ; \stackrel{p+1}{\otimes} T^{*} M \otimes \mathrm{g}_{P}\right)$, for which we use the same symbol $\nabla$, by

$$
\nabla \Phi=\Sigma_{\mu} \Sigma_{\mu_{1}, \cdots, \mu_{p}} \nabla_{\mu} \Phi_{\mu_{1} \cdots \mu_{p}} d x^{\mu} \otimes d x^{\mu_{1}} \otimes d x^{\mu_{2}} \otimes \cdots \otimes d x^{\mu_{p}}
$$




$$
\nabla_{\mu} \Phi_{\mu_{1} \cdots \mu_{p}}=\frac{\partial}{\partial x^{\mu}}\left(\Phi_{\mu_{1} \cdots \mu_{p}}\right)+\left[\Phi_{\mu_{1} \cdots \mu_{p}}, A_{\mu}^{\nabla}\right]
$$

for $\Phi=\sum_{\mu_{1}, \cdots, \mu_{p}} \Phi_{\mu_{1} \cdots \mu_{p}} d x^{\mu_{1}} \otimes \cdots \otimes d x^{\mu_{p}} \in \Gamma\left(M ; \bigotimes^{p} T^{*} M \otimes \mathrm{g}_{P}\right)$. For any $\mathrm{g}_{P}$-valued $p$-form $\Phi=(1 / p !) \sum_{\mu_{1}, \cdots, \mu_{p}} \Phi_{\mu_{1} \cdots \mu_{p}} d x^{\mu_{1}} \wedge \cdots \wedge d x^{\mu_{p}}\left(\Phi_{\mu_{1} \cdots \mu_{p}}\right.$ is skew symmetric with respect to indices $\left.\mu_{1}, \cdots, \mu_{p}\right) d^{\nabla} \Phi$ is written by the aid of the covariant differentiation $\nabla$ by

$$
d^{\nabla} \Phi=\frac{1}{p !} \Sigma_{\mu} \Sigma_{\mu_{1}, \cdots, \mu_{p}} \nabla_{\ell l} \Phi_{\mu_{1} \cdots \mu_{p}} d x^{\mu} \wedge d x^{\mu_{1}} \wedge \cdots \wedge d x^{\mu_{p}} .
$$

We set $\left[\nabla_{\mu}, \nabla_{\nu}\right] \Phi_{\mu_{1} \cdots \mu_{p}}=\nabla_{\mu} \nabla_{\nu} \Phi_{\mu_{1} \cdots \mu_{p}}-\nabla_{\nu} \nabla_{\mu} \Phi_{\mu_{1} \cdots \mu_{p}}$ for $\Phi \in \Gamma\left(M ; \stackrel{p}{\otimes} T^{*} M \otimes \mathrm{g}_{P}\right)$ and we represent the curvature form $R^{\nabla}$ locally by $R^{\nabla}=(1 / 2) \sum_{\mu, \nu} R_{\mu \nu}^{\nabla} d x^{\mu} \wedge d x^{\nu}$, $R^{\nabla_{\mu \nu}}=-R_{\nu \mu}^{\nabla_{\nu}}=\left(\partial / \partial x^{\mu}\right) A^{\nabla_{\nu}}-\left(\partial / \partial x^{\nu}\right) A^{\nabla}{ }_{\mu}-\left[A^{\nabla}{ }_{\mu}, A^{\nabla_{\nu}}\right]$. Then we obtain the Ricci formula which will be used in proving a Bochner's type formula in Section 5;

$$
\left[\nabla_{\mu}, \nabla_{\nu}\right] \Phi_{\mu_{1} \cdots \mu_{p}}=\left[\Phi_{\mu_{1} \cdots \mu_{p}}, R_{\mu \nu}^{\nabla}\right] \text {. }
$$

On each $A^{p}\left(\mathrm{~g}_{P}\right)$ we define an inner product by the aid of Hodge star operator * as follows ;

$$
\langle\Phi, \Psi\rangle_{M}=-\int_{M} \operatorname{Tr}(\Phi \wedge * \Psi), \quad \Phi, \Psi \in A^{p}\left(\mathfrak{g}_{P}\right) .
$$

Since the trace operator $\operatorname{Tr} A \cdot B$ on $\mathfrak{g l}(n ; \mathbb{C})$ is adjoint invariant and its restriction to $\mathrm{g}$ is negative definite, the above global inner product is well defined.

A functional $\mathscr{Y} \mathscr{M}$ on $\mathcal{C}_{E, G}$ defined by

$$
\text { y } \mathscr{M}(\nabla)=\frac{1}{2}\left\|R^{\nabla}\right\|_{M}^{2}, \quad \nabla \in \mathcal{C}_{E, G}
$$

is called Yang-Mills functional.

Definition 1.2. A $G$-connection is called a Yang-Mills $G$-connection if it gives a critical point of $\mathscr{Y} \mathscr{M}$.

We see that a $G$-connection is a Yang-Mills connection if and only if it satisfies a quasi-linear elliptic differential equation of second order $([5]) d^{\nabla}\left(* R^{\nabla}\right)$ $=0$.

The gauge group $\mathcal{G}_{P}$ operates on $\mathcal{C}_{E, G}$ as $f(\nabla)=f^{-1} \circ \nabla \circ f$ for $f \in \mathcal{G}_{P}$ and $\nabla \in$ $\mathcal{C}_{E, G}$. Then the connection form $A^{f(\nabla)}$ and the curvature form $R^{f(\nabla)}$ of $f(\nabla)$ are given by

$$
\left\{\begin{array}{l}
A^{f(\nabla)}=d f \cdot f^{-1}+A d(f) A^{\nabla}, \\
R^{f(\nabla)}=A d(f) R^{\nabla} .
\end{array}\right.
$$

Hence, $\mathscr{Y} \mathscr{M}$ is $\mathcal{G}_{P}$-invariant and for every $f$ of $\mathcal{G}_{P} f(\nabla)$ is a Yang-Mills $G$-connection if so is $\nabla$.

In the following, $G$-connections are identified from the viewpoint of physical meaning if they are transformed by gauge transformations. 
Now suppose that $M$ is real four dimensional. Since * operates on the 2form bundle $\Lambda^{2}$ and satisfies $* * *=i d, \Lambda^{2}$ splits into $\Lambda^{2}=\Lambda_{+}^{2}+\Lambda_{-}^{2}$ with eigenspaces $\Lambda_{ \pm}^{2}$ corresponding to eigenvalues \pm 1 . We extend the operation of $*$ on $\Lambda^{2} \otimes \mathrm{g}_{P}$. Then $R^{\nabla}$ splits into $R^{\nabla}=R_{+}^{\nabla}+R_{-}^{\nabla}, R_{ \pm}^{\nabla} \in \Gamma\left(M ; \Lambda_{ \pm}^{2} \otimes \mathrm{g}_{P}\right)$.

The notion of the (anti-)self-duality of a $G$-connection is given by

Definition 1.3. A $G$-connection $\nabla$ is called (anti-)self-dual if $R^{\nabla}$ satisfies that

$$
R_{-}^{\nabla}=0 \quad\left(R_{+}^{\nabla}=0\right),
$$

respectively.

We see that from Bianchi's identity (1.10) an (anti-)self-dual $G$-connection is a Yang-Mills $G$-connection. Since the first Pontrjagin number $\operatorname{Pont}_{1}(E)$ is given by $-\left(1 / 4 \pi^{2}\right) \int_{M} \operatorname{Tr}\left(R^{\nabla} \wedge R^{\nabla}\right)=\left(1 / 4 \pi^{2}\right)\left(\left\|R_{+}^{\nabla}\right\|_{M}^{2}-\left\|R_{-}^{\nabla}\right\|_{M}^{2}\right)$ from the Chern-Weil theorem and $\mathscr{G} \mathscr{M}(\nabla)=(1 / 2)\left(\left\|R_{+}^{\nabla}\right\|_{M}^{2}+\left\|R_{-}^{\nabla}\right\|_{M}^{2}\right)$, we see that $\mathscr{G} \mathscr{M}(\nabla) \geqq 2 \pi^{2} \mid$ Pont $_{1}(E) \mid$ for all $\nabla \in \mathcal{C}_{E, G}$ and the equality holds if and only if $\nabla$ is (anti-)self-dual. If the bundle $E$ admits an (anti-)self-dual $G$-connection, then $\operatorname{Pont}_{1}(E)$ must be nonnegative (non-positive).

Let $M$ be a compact complex surface with a Hermitian metric $g=$ $2 \sum_{\mu, \nu} g_{\mu \bar{\nu}} d z^{\mu} \cdot d \bar{z}^{\nu}$. We use the following notation for the complex surface $M$. We let $\Lambda_{c}^{k}$ and $\Lambda^{p, q}$ denote the complexification of the $k$-form bundle $\Lambda^{k}$ and the bundle consisting of complex forms of type $(p, q)$, respectively. And by $A_{c}^{k}$ and $A^{p, q}$ we denote $\Gamma\left(M ; \Lambda_{c}^{k}\right)$ and $\Gamma\left(M ; \Lambda^{p, q}\right)$, respectively. Moreover, for a complex vector bundle $F \Gamma\left(M ; \Lambda_{c}^{k} \otimes F\right)$ and $\Gamma\left(M ; \Lambda^{p, q} \otimes F\right)$ are denoted by $A_{C}^{k}(F)$ and $A^{p, q}(F)$, respectively. Then we see that $\Lambda_{C}^{k}=\Sigma_{p+q=k} \Lambda^{p, q}, A_{C}^{k}=\Sigma_{p+q=k} A^{p, q}$ and $A_{C}^{k}(F)=\sum_{p+q=k} A^{p, q}(F)$.

Let $\mathrm{g}^{C}$ be the complexification of $\mathrm{g}$ and - the conjugation on $\mathrm{g}^{C}$ with respect to $\mathrm{g}$. Then the complexification of real bundle $g_{P}$ coincides with $P \times_{A d} g^{C}$ which we denote by $g_{P}^{C}$. By the aid of the conjugation - on $g^{c}$ together with the natural conjugation - on $\Lambda_{c}^{k}$ we define the conjugation - on $\Lambda_{c}^{k} \otimes \mathrm{g}_{P}^{c}$ such that $\bar{\Phi} \in \Lambda^{q, p} \otimes \mathrm{g}_{P}^{c}$ for $\Phi \in \Lambda^{p, q} \otimes \mathrm{g}_{P}^{c}$. Notice that a $\mathrm{g}_{P}^{c}$-valued $k$-form $\Phi$ takes vaues in $\mathrm{g}_{P}$ if and only if $\bar{\Phi}=\Phi$.

For a $G$-connection $\nabla$ the covariant derivative $\nabla \Phi$ of $\Phi \in A_{C}^{0}\left(\mathrm{~g}_{P}^{C}\right)$ belongs to $A_{C}^{1}\left(\mathrm{~g}_{P}^{C}\right)=A^{1,0}\left(\mathrm{~g}_{P}^{C}\right)+A^{0,1}\left(\mathrm{~g}_{P}^{C}\right)$. We denote by $\nabla^{+} \Phi$ and $\nabla^{-} \Phi(1,0)$-part and $(0,1)-$ part of $\nabla \Phi$, respectively. Moreover, $d^{\nabla}$ splits into $d^{\nabla}=\partial^{\nabla}+\bar{\partial}^{\nabla}$ on $A^{p, q}\left(\mathrm{~g}_{P}^{C}\right)$ as follows ;

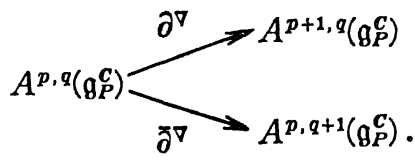


The operators $\partial^{\nabla}$ and $\bar{\partial}^{\nabla}$ have the following expressions;

$$
\left\{\begin{array}{l}
\left(\partial^{\nabla} \Phi\right)^{i}{ }_{j}=\partial\left(\Phi^{i}{ }_{j}\right)+(-1)^{p+q}\left[\Phi \wedge A^{\nabla+}\right]_{j}^{i} \\
\left(\bar{\partial}^{\nabla} \Phi\right)^{i}{ }_{j}=\bar{\partial}\left(\Phi^{i}{ }_{j}\right)+(-1)^{p+q}\left[\Phi \wedge A^{\nabla-}\right]_{j}^{i}{ }_{j}
\end{array}\right.
$$

for $\Phi=\sum_{i, j} \Phi^{i} t_{i} \otimes t^{j} \in A^{p, q}\left(\mathrm{~g}_{P}^{C}\right)$, where $A^{\nabla+}$ and $A^{\nabla-}$ are $(1,0)$-part and $(0,1)$-part of the connection form $A^{\nabla}$, respectively.

\section{§2. Deformations of Anti-Self-Dual Connections}

In this section we shall discuss the space of infinitesimal deformations of irreducible anti-self-dual $G$-connections and we shall show that this space is nothing but the first cohomology group $H^{1}$ of an elliptic complex associated with a fixed irreducible anti-self-dual $G$-connection (Proposition 2.4).

First we show a proposition which characterizes an anti-self-dual $G$-connection. Before proving this proposition we need a key lemma which gives a characterization of an (anti-)self-dual form in terms of complex forms of type $(p, q)$.

We fix an orientation on $M$ induced from the complex structure of $M$.

An inner product $\langle\cdot, \cdot\rangle$ induced from $g$ on the cotangent space $T^{*} M=\Lambda^{1}$ at $x$ is given by the aid of a local holomorphic coordinate $\left\{z^{\mu}\right\}_{\mu=1,2}$ as follows;

$$
\langle\sigma, \tau\rangle=\sum_{\mu, \nu} g^{\mu \bar{\nu}}(x)\left(\sigma_{\mu} \bar{\tau}_{\nu}+\tau_{\mu} \bar{\sigma}_{\nu}\right)
$$

for $\sigma=\Sigma_{\mu}\left(\sigma_{\mu} d z^{\mu}+\bar{\sigma}_{\mu} d \bar{z}^{\mu}\right), \tau=\Sigma_{\mu}\left(\tau_{\mu} d z^{\mu}+\bar{\tau}_{\mu} d \bar{z}^{\mu}\right) \in \Lambda_{x}^{1}$, where $\left(g^{\mu \bar{\nu}}(x)\right)$ denotes the inverse matrix of $\left(g_{\mu \bar{\nu}}(x)\right)$. We choose a suitable local holomorphic coordinate $\left\{z^{1}=x^{1}+\sqrt{-1} x^{2}, z^{2}=x^{3}+\sqrt{-1} x^{4}\right\}$ around $x$ such that $g_{\mu \bar{\nu}}(x)=\delta_{\mu \nu}$. Then we have that $\left\langle d x^{\mu}, d x^{\nu}\right\rangle=(1 / 2) \delta^{\mu \nu}$ for real 1 -forms $d x^{\mu}, 1 \leqq \mu \leqq 4$.

Since the orientation is given by $\left\{d x^{1}, \cdots, d x^{4}\right\}$, we see that the following 2 -forms constitute a basis of $\Lambda_{+}^{2}$ at $x$;

$$
\left\{\begin{array}{l}
d x^{1} \wedge d x^{2}+d x^{3} \wedge d x^{4}=\frac{\sqrt{-1}}{2}\left(d z^{1} \wedge d \bar{z}^{1}+d z^{2} \wedge d \bar{z}^{2}\right) \\
d x^{1} \wedge d x^{3}-d x^{2} \wedge d x^{4}=\frac{1}{2}\left(d z^{1} \wedge d z^{2}+d \bar{z}^{1} \wedge d \bar{z}^{2}\right) \\
d x^{1} \wedge d x^{4}+d x^{2} \wedge d x^{3}=\frac{-\sqrt{-1}}{2}\left(d z^{1} \wedge d z^{2}-d \bar{z}^{1} \wedge d \bar{z}^{2}\right)
\end{array}\right.
$$

Because the fundamental form $\Omega=\sqrt{-1} \Sigma_{\mu, \nu} g_{\mu \bar{\nu}} d z^{\mu} \wedge d \bar{z}^{\nu}$, associated to $g$ is reduced to $\Omega=\sqrt{-1}\left(d z^{1} \wedge d \bar{z}^{1}+d z^{2} \wedge d \bar{z}^{2}\right)$ at $x$, it is observed from (2.2) that these 2 -forms are written by $\beta+\bar{\beta}+a \Omega$ for a form of type $(2,0) \beta$ and a real $a$. Similarly we see that the following 2 -forms which give a basis of $\Lambda_{-}^{2}$ at $x$ are forms of type $(1,1)$, orthogonal to $\Omega$; 


$$
\left\{\begin{array}{l}
d x^{1} \wedge d x^{2}-d x^{3} \wedge d x^{4}=\frac{\sqrt{-1}}{2}\left(d z^{1} \wedge d \bar{z}^{1}-d z^{2} \wedge d \bar{z}^{2}\right) \\
d x^{1} \wedge d x^{3}+d x^{2} \wedge d x^{4}=\frac{1}{2}\left(d z^{1} \wedge d \bar{z}^{2}-d z^{2} \wedge d \bar{z}^{1}\right) \\
d x^{1} \wedge d x^{4}-d x^{2} \wedge d x^{3}=\frac{\sqrt{-1}}{2}\left(d z^{1} \wedge d \bar{z}^{2}+d z^{2} \wedge d \bar{z}^{1}\right)
\end{array}\right.
$$

Here the inner product $\langle\cdot, \cdot\rangle$ on $\Lambda_{R}^{1,1}=\left\{\alpha \in \Lambda^{1,1}, \bar{\alpha}=\alpha\right\}$ is given at $x$ by

$$
\langle\alpha, \gamma\rangle=\sum_{\mu, \nu, \tau, \varepsilon} g^{\mu \bar{\nu}}(x) g^{\nu \bar{\varepsilon}}(x) \alpha_{\mu \bar{\nu}} \overline{\gamma_{\tau \bar{\varepsilon}}}=\sum_{\mu, \nu} \alpha_{\mu \nu} \overline{\gamma_{\mu \bar{\nu}}}
$$

for $\alpha=\sum_{\mu, \nu} \alpha_{\mu \bar{\nu}} d z^{\mu} \wedge d \bar{z}^{\nu}$ and $\gamma=\Sigma_{\mu, \nu} \gamma_{\mu \bar{\nu}} d z^{\mu} \wedge d \bar{z}^{\nu}$ of $\Lambda_{R}^{1,1}$ at $x$.

Since that a form is of type $(p, q)$ is free of holomorphic local coordinates, we obtain the following

Lemma 2.1 ([2]). (i) A real 2-form $\alpha$ is self-dual if and only if $\alpha$ is written by $\alpha=\alpha^{2,0}+\overline{\alpha^{2,0}}+a \Omega$ for a form of type $(2,0) \alpha$ and a real number $a$, and

(ii) a real 2-form $\alpha$ is anti-self-dual if and only if $\alpha$ is a real form of type $(1,1)$, orthogonal to $\Omega$.

Remark. (i) Because $\|\Omega\|^{2}=2, \Omega$ is a nowhere vanishing section of $\Lambda_{\boldsymbol{R}}^{1,1}$. Then from this lemma the complexification of $\Lambda_{+}^{2}$ is represented by $\left(\Lambda_{+}^{2}\right)_{C}=$ $K \oplus \bar{K} \oplus \mathbb{C} \Omega$ as a Whitney sum, where $K$ and $\bar{K}$ are the canonical line bundle of $M$ and its conjugate bundle, respectively.

(ii) The complexification $\left(\Lambda_{-}^{2}\right)_{c}$ of $\Lambda_{-}^{2}$ consists of primitive forms of type $(1,1)$. Here a form $\alpha$ is primitive if $\alpha$ satisfies $\Omega \wedge * \alpha=0$ ([9] and [14]).

We extend $\langle\cdot, \cdot\rangle$ on $\Lambda_{R}^{1,1}$ to a bilinear mapping $\langle\cdot, \cdot\rangle: \Lambda_{R}^{1,1} \otimes \mathrm{g}_{P} \times \Lambda_{R}^{1,1} \rightarrow \mathrm{g}_{P}$. Then as an immediate consequence of Lemma 2.1, we have

Proposition 2.2. A G-connection $\nabla$ is anti-self-dual if and only if $R^{\nabla}$ is a $\mathrm{g}_{P}$-valued form of type $(1,1)$, orthogonal to $\Omega$, that $i$, $R^{\nabla}$ belongs to $A^{1,1}\left(\mathrm{~g}_{P}^{C}\right)$ and satisfies that $\overline{R^{7}}=R^{\nabla}$ and $\left\langle R^{\nabla}, \Omega\right\rangle=0$.

Since $R^{\nabla}$ of an anti-self-dual $G$-connection $\nabla$ is a form of type $(1,1)$, the following is derived from (1.11) for $\Phi \in A^{p, q}\left(\mathrm{~g}_{P}^{C}\right)$;

$$
\partial^{\nabla} \circ \partial^{\nabla} \Phi=0, \quad \bar{\partial}^{\nabla} \circ \bar{\partial}^{\nabla} \Phi=0 \quad \text { and }\left(\partial^{\nabla} \circ \bar{\partial}^{\nabla}+\bar{\partial}^{\nabla} \circ \partial^{\nabla}\right) \Phi=\left[\Phi \wedge R^{\nabla}\right] .
$$

Note. Since the image of $G$ by $\rho$ in $G L(n, C)$ is a compact subgroup of $S U(n)$ for a certain frame $\left\{e_{i}\right\}$ of $\mathbb{C}^{n}, E$ admits a Hermitian structure $h$ induced from the $G$-structure. The structure $h$ is preserved by all $\nabla \in \mathcal{C}_{E, G}$. If a $G$ connection $\nabla$ is anti-self-dual, then from Theorem 5.1 in [2] $\nabla$ induces a holomorphic structure $J$ on $E$ such that $\nabla$ is a unique holomorphic Hermitian connection, that is, $\nabla h=0$ and $\nabla J=0$.

Now suppose that $\nabla^{t}(|t|<\varepsilon)$ is a one-parameter family of anti-self-dual $G$ - 
connections on $E$ with $\nabla^{0}=\nabla$. Since the infinitesimal deformation $B=\left.(d / d t) \nabla^{t}\right|_{t=0}$ of $\nabla^{t}$ at $t=0$ defines an element of $A^{1}\left(\mathrm{~g}_{P}\right)$, it follows from (1.9) that the curvature form $R^{\nabla^{t}}=d A^{\nabla^{t}}-A^{\nabla^{t}} \wedge A^{\nabla^{t}}$ is reduced to

$$
R^{\nabla^{t}}=R^{\nabla}+t d^{\nabla} B+o(t)
$$

for a $g_{P}$-valued 2-form $o(t)$ of small order with respect to $t$.

Since $B$ splits into $B=B^{+}+\overline{B^{+}}$for $B^{+} \in A^{1,0}\left(\mathrm{~g}_{P}^{C}\right), d^{\nabla} B=\partial^{\nabla} B^{+}+\left(\partial^{\nabla} \overline{B^{+}}+\bar{\partial}^{\nabla} B^{+}\right)$ $+\bar{\partial}^{\nabla} \overline{B^{+}}$. Then from Proposition 2.2 the infinitesimal deformation $B$ satisfies that

$$
\partial^{\nabla} B^{+}=0, \quad \bar{\partial}^{\nabla} \overline{B^{+}}=0 \quad \text { and }\left\langle\partial^{\nabla} \overline{B^{+}}+\bar{\partial}^{\nabla} B^{+}, \Omega\right\rangle=0 .
$$

We define $d_{+}^{\nabla}$ from $A^{1}\left(\mathrm{~g}_{P}\right)$ to $A_{+}^{2}\left(\mathrm{~g}_{P}\right)=\Gamma\left(M ; \Lambda_{+}^{2} \otimes \mathrm{g}_{P}\right)$, which is well defined from Remark (i) of Lemma 2.1, by

$$
d \underset{+}{\nabla}\left(B^{+}+\overline{B^{+}}\right)=\partial^{\nabla} B^{+}+\Omega \otimes\left\langle\partial^{\nabla} \overline{B^{+}}+\bar{\partial}^{\nabla} B^{+}, \Omega\right\rangle+\bar{\partial}^{\nabla} \overline{B^{+}}
$$

for $B^{+} \in A^{1,0}\left(\mathrm{~g}_{P}^{C}\right)$. Then we obtain the following

Proposition 2.3. $A g_{P}$-valued 1-form $B$ gives an infinitesimal deformation of an anti-self-dual $G$-connection $\nabla$ preserving anti-self-duality if and only if $B$ belongs to $\operatorname{Ker} d_{+}^{\nabla}$.

Now we let $f_{t}(|t|<\varepsilon)$ be a one-parameter family of gauge transformations of $P$ with $f_{0}=i d e n t i t y$. Then the differential of $f_{t}$ at $t=0, \dot{f}=\left.(d / d t) f_{t}\right|_{t=0}$, gives a smooth section of $\mathrm{g}_{P}$, that is, $\dot{f} \in A^{0}\left(\mathrm{~g}_{P}\right)$. Notice that conversely every smooth section $f$ of $\mathrm{g}_{P}$ induces a one-parameter subgroup $f_{t}=\exp (t f)$ of $\mathcal{G}_{P}$. By (1.22) the connection form $A^{f_{t}(\nabla)}$ of $G$-connection $f_{t}(\nabla)$ satisfies $A^{f(\nabla)}=d f_{t} \cdot\left(f_{t}\right)^{-1}+A d\left(f_{t}\right) A^{\nabla}$. Since $f_{t}=i d+t \dot{f}+o(t)$, it follows that $A^{f_{t}(\nabla)}=A^{\nabla}+t \nabla \dot{f}+o(t)$, that is, $\nabla \dot{f}$ gives an infinitesimal deformation of $\nabla$. Because $f_{t}(\nabla)$ is anti-self-dual, we see from Proposition 2.3 that $\nabla \dot{f}$ is in $\operatorname{Ker} d_{+}^{\nabla}$.

Proposition 2.4. The space of infinitesimal deformations of an anti-self-dual $G$-connection $\nabla$ preserving anti-self-duality, that is, the tangent space of $\mathscr{M}_{E, G}^{-}$at $\nabla$ coincides with the first cohomology group $H^{1}=\operatorname{Ker} d_{+}^{\nabla} / \operatorname{Im} \nabla$ of elliptic complex

$$
0 \longrightarrow A^{0}\left(\mathrm{~g}_{P}\right) \stackrel{{ }^{d(0)}=\nabla}{\longrightarrow} A^{1}\left(\mathrm{~g}_{P}\right) \stackrel{d^{(1)}=d_{+}^{\nabla}}{\longrightarrow} A_{+}^{2}\left(\mathrm{~g}_{P}\right) \longrightarrow 0 .
$$

Proof. In order to verify that (2.8) is elliptic we show that the symbol sequence of (2.8) is exact ([1]).

By the aid of (1.24) and (2.7), the symbol sequence extended to the complex numbers $C$ is

$$
0 \longrightarrow p^{*}\left(\Lambda_{C}^{0} \otimes \mathrm{g}_{P}^{C}\right) \stackrel{\sigma(d) \otimes i d_{\mathrm{g}}^{C}}{\longrightarrow} p^{*}\left(\Lambda_{C}^{1} \otimes \mathrm{g}_{P}^{C}\right) \stackrel{\sigma\left(d_{+}\right) \otimes i d_{\mathrm{g}}^{C}}{\longrightarrow} p^{*}\left(\left(\Lambda_{+}^{2}\right)_{c} \otimes \mathrm{g}_{P}^{C}\right) \longrightarrow 0,
$$

where $p$ is the projection of $T^{*} M \backslash\{0\}$ to $M, \sigma(d)$ and $\sigma\left(d_{+}\right)$are the principal symbols of the following elliptic complex associated to $M$; 


$$
0 \longrightarrow A_{C}^{0} \stackrel{d}{\longrightarrow} A_{C}^{1} \stackrel{a_{+}}{\longrightarrow}\left(A_{+}^{2}\right)_{c}=\Gamma\left(M ;\left(\Lambda_{+}^{2}\right)_{C}\right) \longrightarrow 0 \text { 。 }
$$

Here $d$ is the exterior differentiation and $d_{+}$is defined by $d_{+}\left(\tau^{+}+\tau^{-}\right)=\partial \tau^{+}+$ $\left\langle\bar{\partial} \tau^{+}+\partial \tau^{-}, \Omega\right\rangle \Omega+\bar{\partial} \tau^{-}$for $\tau^{+} \in A^{1,0}$ and $\tau^{-} \in A^{0,1}$. Thus the exactness of (2.9) is an immediate consequence of the ellipticity of (2.10).

\section{§3. Vanishing Theorem}

We set $D^{(0)}=d^{(0) *} \circ d^{(0)}, D^{(1)}=d^{(1) *} \circ d^{(1)}+d^{(0)} \circ d^{(0) *}$ and $D^{(2)}=d^{(1)} \circ d^{(1) *}$ for the complex (2.8). Since (2.8) is elliptic, each $D^{(i)}$ is an elliptic operator and each $i$-th cohomology group $H^{i}=\operatorname{Ker} d^{(i)} / \operatorname{Im} d^{(i-1)}$ is isomorphic to $\operatorname{Ker} D^{(i)}, i=0,1$ and 2. Here $d^{(i) *}$ is the formal adjoint operator of $d^{(i)}$ with respect to the inner products $\langle, \cdot,\rangle_{M}$ given at $(1.20)$.

From the Atiyah-Singer index theorem the index $h^{0}-h^{1}+h^{2}\left(h^{i}=\operatorname{dim} H^{i}\right.$, $i=0,1,2)$ can be represented in terms of the characteristic classes of $M$ and the Lie algebra bundle $\mathrm{g}_{P}$. Therefore to obtain the dimension of the space of infinitesimal deformations of an irreducible anti-self-dual $G$-connection $\nabla$ it suffices to estimate $h^{0}$ and $h^{2}$.

Since $\nabla$ is irreducible, it is easily verified that $h^{0}=0$ in the following manner. Assume that $\Phi \in A^{0}\left(\mathfrak{g}_{P}\right)$ satisfies $D^{(0)} \Phi=0$. Then we see that $\|\nabla \Phi\|_{M}^{2}=\left\langle D^{(0)} \Phi, \Phi\right\rangle_{M}$ $=0$, that is, $\Phi$ is parallel. Hence $\Phi$ commutes with the holonomy group of $\nabla$, which is an open subgroup of $G$. That $\Phi=0$ follows from the semi-simplicity of $G$.

Now we suppose that $M$ is a Kähler surface. Then we have the following vanishing theorem.

Theorem 3.1. Let $M$ be a compact Kähler surface with a Kähler metric $g$ of positive scalar curvature. Then $h^{2}=0$ for each irreducible anti-self-dual $G$ connection $\nabla$.

This theorem is verified by the aid of the following key lemmas, whose proof will be shown in Section 5 .

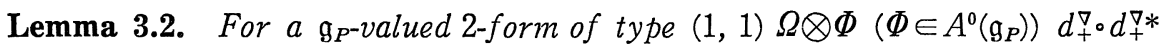
$(\Omega \otimes \Phi)$ is written by

$$
d_{+}^{\nabla} \cdot d_{+}^{\nabla *}(\Omega \otimes \Phi)=-4 \sqrt{-1} \Omega \otimes\left\langle\partial^{\nabla} \circ \bar{\partial}^{\nabla} \Phi, \Omega\right\rangle .
$$

Lemma 3.3. Every $\mathrm{g}_{P}^{C}$-valued 2-form of type $(2,0) \Phi=(1 / 2) \Sigma_{\mu, \nu} \Phi_{\mu \nu} d z^{\mu} \wedge d z^{\nu}$ $\left(\Phi_{\mu \nu}=-\Phi_{\nu \mu}\right)$ satisfies that

$$
\left\{\left(\partial^{\nabla} \circ \partial^{\nabla *}+\partial^{\nabla *} \circ \partial^{\nabla}\right) \Phi\right\}_{\beta \gamma}=-\Sigma_{\alpha, \mu} g^{\alpha \bar{\mu}} \tilde{\nabla}_{\bar{\mu}} \tilde{\nabla}_{\alpha} \Phi_{\beta \gamma}+\frac{1}{2} \rho \Phi_{\beta \gamma} \cdot
$$

Here $\partial^{\nabla *}$ is the formal adjoint of $\partial^{\nabla}$ with respect to inner products $\langle\cdot, \cdot\rangle_{M}$ on $A^{p, 0}\left(\mathrm{~g}_{P}^{C}\right), p=1,2$ given at (3.4) later and $\rho$ is the scalar curvature of the 
metric $g$. And we denote by $\tilde{\nabla}$ the sum of the Levi-civita Connection $D$ on $A^{p, 0}$ and $\nabla$ on $\Gamma\left(M ; g_{P}^{C}\right)$, that is, the covariant derivative $\tilde{\nabla}_{\mu}$ is given for example by

$$
\tilde{\nabla}_{\mu} \Phi_{\nu}=D_{\mu} \Phi_{\nu}+\left[\Phi_{\nu}, A_{\mu}^{\nabla+}\right]=\frac{\partial}{\partial z^{\mu}}\left(\Phi_{\nu}\right)-\sum_{\sigma} \Gamma_{\mu \nu}^{\sigma} \Phi_{\sigma}+\left[\Phi_{\nu}, A_{\mu}^{\nabla^{+}}\right]
$$

by the aid of the Christoffel symbols $\Gamma_{\mu \nu}^{\sigma}$ of $D$ and the coefficients $A_{\mu}^{\nabla^{+}}$of $A^{\nabla}$.

Note. From Lemma 3.2, $d_{+}^{\nabla} \cdot d_{+}^{\nabla *}(\Omega \otimes \Phi)$ is again a form of type $(1,1)$ for any $\Omega \otimes \Phi$.

Before proving Theorem 3.1, we give integral expressions of the global inner products $\langle\cdot, \cdot\rangle_{M}$ on $A^{1}\left(\mathrm{~g}_{P}\right)$ and $A_{+}^{2}\left(\mathrm{~g}_{P}\right)$ by introducing inner products $\langle\cdot, \cdot\rangle_{M}$ on $A^{p, 0}\left(\mathrm{~g}_{P}^{C}\right), p=1,2$. We define the inner products $\langle\cdot, \cdot\rangle_{M}$ on $A^{p, 0}\left(\mathrm{~g}_{P}^{C}\right)$ by

for

$$
\begin{gathered}
\left\langle\Phi^{p, 0}, \Psi^{p, 0}\right\rangle_{M}=\int_{M}\left\langle\Phi^{p, 0}, \Psi^{p, 0}\right\rangle d v, \\
\left\langle\Phi^{p, 0}, \Psi^{p, 0}\right\rangle=\frac{-1}{p !} \sum_{\substack{\mu_{1}, \cdots, \mu_{p} \\
\nu_{1}, \cdots, p}} g^{\mu_{1} \bar{\nu}_{1} \cdots g^{\mu} g^{\bar{\nu}} p} \operatorname{Tr}\left(\Phi_{\mu_{1} \cdots \mu_{p}}^{p, 0} \cdot \overline{\Psi_{\nu_{1}, \nu_{p}}^{p, 0}}\right)
\end{gathered}
$$

$$
\begin{aligned}
& \Phi^{p, 0}=\frac{1}{p !} \sum_{\mu} \Phi_{\mu_{1} \cdots \mu_{p}}^{p_{0}, 0} d z^{\mu_{1}} \wedge \cdots \wedge d z^{\mu_{p}}, \\
& \Psi^{p, 0}=\frac{1}{p !} \sum_{\mu} \Psi_{\mu_{1} \cdots \mu_{p}}^{p_{0}, 0} d z^{\mu_{1}} \wedge \cdots \wedge d z^{\mu} p \in A^{p, 0}\left(\mathrm{~g}_{P}^{C}\right),
\end{aligned}
$$

where $d v$ is the volume element induced from $g$. By the aid of (3.4) the inner products $\langle\cdot, \cdot\rangle_{M}$ on $A^{1}\left(\mathrm{~g}_{P}\right)$ and $A_{+}^{2}\left(\mathrm{~g}_{P}\right)$ are written by

$$
\begin{aligned}
& \left\langle\Phi^{1}, \Psi^{1}\right\rangle_{M}=\int_{M}\left\langle\Phi^{1}, \Psi^{1}\right\rangle d v, \\
& \left\langle\Phi^{1}, \Psi^{1}\right\rangle=2 \operatorname{Re}\left\langle\Phi^{1^{+}}, \Psi^{1^{+}}\right\rangle
\end{aligned}
$$

for $\Phi^{1}=\Phi^{1^{+}}+\overline{\Phi^{1^{+}}}$and $\Psi^{1}=\Psi^{1^{+}}+\overline{\Psi^{1^{+}}}\left(\Phi^{1^{+}}, \Psi^{1^{+}} \in A^{1,0}\left(\mathrm{~g}_{P}^{C}\right)\right)$ and

$$
\begin{gathered}
\left\langle\Phi^{2}, \Psi^{2}\right\rangle_{M}=\int_{M}\left\langle\Phi^{2}, \Psi^{2}\right\rangle d v, \\
\left\langle\Phi^{2}, \Psi^{2}\right\rangle=2 \operatorname{Re}\left\langle\Phi^{2,0}, \Psi^{2,0}\right\rangle+2\left\langle\Phi^{0}, \Psi^{0}\right\rangle
\end{gathered}
$$

for $\mathrm{g}_{P}$-valued self-dual 2 -forms $\Phi^{2}=\Phi^{2,0}+\overline{\Phi^{2,0}}+\Omega \otimes \Phi^{0}$ and $\Psi^{2}=\Psi^{2,0}+\overline{\Psi^{2,0}}+\Omega \otimes \Psi^{0}$ $\left(\Phi^{2,0}, \Psi^{2,0} \in A^{2,0}\left(\mathfrak{g}_{P}^{C}\right), \Phi^{0}, \Psi^{0} \in A^{0}\left(\mathfrak{g}_{P}\right)\right)$.

Proof of Theorem 3.1. Let $\Psi=\Psi^{2,0}+\overline{\Psi^{2,0}}+\Omega \otimes \Psi^{0} \quad\left(\Psi^{2,0} \in A^{2,0}\left(\mathrm{~g}_{P}^{\boldsymbol{C}}\right), \quad \Psi^{0} \in\right.$ $\left.A^{0}\left(\mathfrak{g}_{P}\right)\right)$ satisfies that $D^{(2)} \Psi=0$. If we take the inner product of $D^{(2)} \Psi$ and $\Psi^{2,0}+\overline{\Psi^{2,0}}$, then we have

$$
\begin{aligned}
0 & =\left\langle D^{(2)} \Psi, \Psi^{2,0}+\overline{\Psi^{2,0}}\right\rangle \\
& =\left\langle d_{+}^{\nabla} d_{+}^{\nabla *}\left(\Psi^{2,0}+\overline{\Psi^{2,0}}\right), \Psi^{2,0}+\overline{\Psi^{2,0}}\right\rangle \\
& =\left\langle\partial^{\nabla} \circ \partial^{\nabla *} \Psi^{2,0}, \Psi^{2,0}\right\rangle+\left\langle\bar{\partial}^{\nabla} \circ \bar{\partial}^{\nabla * \overline{\Psi^{2,0}}}, \overline{\Psi^{2,0}}\right\rangle,
\end{aligned}
$$

where we used the fact that $d_{+}^{\nabla} d_{+}^{\nabla *}\left(\Omega \otimes \Psi^{0}\right)$ is of type $(1,1)$ and that $(1,0)$-part 
of $d_{+}^{\nabla * \Psi^{2,0}}$ is $\partial^{\nabla *} \Psi^{2,0}$, and also (2.7).

Since $M$ is complex two dimensional, $\partial^{\nabla} \Psi^{2,0} \in A^{3,0}\left(\mathrm{~g}_{P}^{C}\right)=\{0\}$ and also $\bar{\partial}^{\nabla} \overline{\Psi^{2,0}}$ $\in A^{0,3}\left(\mathrm{~g}_{P}^{C}\right)=\{0\}$. It follows that

$$
0=\left\langle\left(\partial^{\nabla} \circ \partial^{\nabla *}+\partial^{\nabla *} \partial^{\nabla}\right) \Psi^{2,0}, \Psi^{2,0}\right\rangle+\left\langle\left(\bar{\partial}^{\nabla} \circ \bar{\partial}^{\nabla} *+\bar{\partial}^{\nabla} \circ \bar{\partial}^{\nabla}\right) \overline{\Psi^{2,0}}, \overline{\Psi^{2,0}}\right\rangle .
$$

Because $\bar{\partial}^{\nabla} \circ \bar{\partial}^{\nabla *}+\bar{\partial}^{\nabla *} \circ \bar{\partial}^{\nabla}=\left(\partial^{\nabla} \circ \partial^{\nabla *}++\partial^{\nabla} \cdot \partial^{\nabla}\right)^{-}$, the above is reduced to

$$
0=\operatorname{Re}\left\langle\left(\partial^{\nabla} \circ \partial^{\nabla *}+\partial^{\nabla *} \circ \partial^{\nabla}\right) \Psi^{2,0}, \Psi^{2,0}\right\rangle .
$$

We apply Lemma 3.3 to (3.8). Then we see that

$$
\begin{aligned}
0=\operatorname{Re}\{ & \left\{\sum_{\substack{\alpha, \beta, \sigma, \tau \\
\nu, \mu}}, g^{\mu \bar{\sigma}} g^{\nu \bar{z}} g^{\alpha \bar{\beta}} \operatorname{Tr}\left(\tilde{\nabla}_{\bar{\beta}} \tilde{\nabla}_{\alpha} \Psi_{\mu \nu}^{2,0}\right) \cdot \overline{\Psi_{\sigma \tau}^{2,0}}\right. \\
& \left.-\frac{1}{2} \sum_{\mu, \nu, \sigma, \tau} g^{\mu \bar{\sigma}} g^{\nu \bar{\tau}} \rho \operatorname{Tr} \Psi_{\mu \nu}^{2,0} \cdot \overline{\Psi_{\sigma \tau}^{2,0}}\right\} .
\end{aligned}
$$

The second term of (3.9) is reduced to $\rho\left\langle\Psi^{2,0}, \Psi^{2,0}\right\rangle$, which is a real number.

Integrate (3.9) over $M$ and use Stokes' lemma. Then we have the following Bochner type formula;

$$
0=\left\langle\nabla^{+} \Psi^{2,0}, \nabla^{+} \Psi^{2,0}\right\rangle_{M}+\int_{M} \rho\left\langle\Psi^{2,0}, \Psi^{2,0}\right\rangle d v
$$

Since the scalar curvature $\rho$ is positive, it follows that $\Psi^{2,0}=0$. If we now apply Lemma 3.2 to $\Psi=\Omega \otimes \Psi^{\circ}$, then

$$
\begin{aligned}
0 & =\left\langle d_{+}^{\nabla} \circ d_{+}^{\nabla *}\left(\Omega \otimes \Psi^{0}\right), \Omega \otimes \Psi^{0}\right\rangle \\
& =8 \operatorname{Tr}\left(\Sigma_{\mu, \nu} g^{\mu \bar{\nu}}\left(\partial^{\nabla} \circ \bar{\partial}^{\nabla} \Psi^{0}\right)_{\mu \bar{\nu}} \cdot \Psi^{0} .\right.
\end{aligned}
$$

We integrate (3.11) over $M$ and use the formula $\left(\partial^{\nabla} \circ \bar{\partial}^{\nabla} \Psi^{0}\right)_{\mu \bar{\nu}}=\tilde{\nabla}_{\mu} \tilde{\nabla}_{\bar{\nu}} \Psi^{0}$. Then we have that

$$
0=8\left\langle\nabla^{+} \Psi^{0}, \nabla^{+} \Psi^{0}\right\rangle_{M},
$$

that is, $\nabla \Psi^{0}=\nabla^{+} \Psi^{0}+\overline{\nabla^{+} \Psi^{0}}=0$. That $\Psi^{0}=0$ follows by the aid of the same argument as one in the proof of $h^{0}=0$. Thus the theorem is proved.

\section{$\S 4$. The Index of the Complex}

Since the symbol sequence (2.9) of the complex (2.8) is given by the symbol sequence of (2.10) being tensored with $i d$ on $g_{P}^{C}$, the index of (2.8) can be calculated by the aid of Proposition 2.17 in [3] as follows;

$$
h^{0}-h^{1}+h^{2}=\frac{\operatorname{ch}\left(\mathrm{g}_{P}^{C}\right)\left\{c h\left(\Lambda_{C}^{0}\right)-c h\left(\Lambda_{C}^{1}\right)+c h\left(\Lambda_{+}^{2}\right)_{c}\right\}}{e(T M)} \times I(T M \otimes C)[M]
$$

here $e(T M)$ is the Euler class of $M, c h(F)$ and $\mathscr{I}(F)$ are the Chern character and the Todd class of a complex vector bundle $F$, respectively.

Theorem 4.1. The index of (2.8) is equal to 


$$
\operatorname{Pont}_{1}\left(\mathrm{~g}_{P}^{C}\right)+\frac{1}{2} \operatorname{dim} G(\chi+\tau) \text {. }
$$

Proof. We suppose that the first Chern class $c_{1}$ of the holomorphic tangent bundle $T^{1,0} M$ splits into $c_{1}\left(T^{1,0} M\right)=x_{1}+x_{2}$. Then we see that $e(T M)=x_{1} x_{2}$, $\operatorname{ch}\left(\Lambda_{\boldsymbol{C}}^{1}\right)=e^{x_{1}}+e^{x_{2}}+e^{-x_{1}}+e^{-x_{2}}$ and $\operatorname{ch}\left(\Lambda_{+}^{2}\right)_{C}=1+e^{\left(x_{1}+x_{2}\right)}+e^{-\left(x_{1}+x_{2}\right)}$. The last formula is derived from Remark (i) of Lemma 2.1. If we apply the Todd class formula;

$$
I(T M \otimes C)=\frac{x_{1} \cdot x_{2}}{\left(1-e^{-x_{1}}\right)\left(1-e^{-x_{2}}\right)} \cdot \frac{\left(-x_{1}\right) \cdot\left(-x_{2}\right)}{\left(1-e^{x_{1}}\right)\left(1-e^{x_{2}}\right)}
$$

to the right-hand side of (4.1), then the index is reduced to

$$
\begin{gathered}
\left\{\operatorname{dim} G+\frac{1}{2}\left(c_{1}^{2}-2 c_{2}\right)\left(\mathrm{g}_{P}^{C}\right)\right\}\left\{2+\frac{1}{6}\left(c_{1}^{2} M+c_{2} M\right)\right\}[M] \\
=\text { Pont }_{1}\left(\mathrm{~g}_{P}^{C}\right)+\frac{1}{6} \operatorname{dim} G\left(c_{1}^{2} M+c_{2} M\right)[M] .
\end{gathered}
$$

Then (4.1) is obtained, if we apply $\chi+\tau=(1 / 3)\left(c_{1}^{2} M+c_{2} M\right)[M]$ to (4.3).

Remark. In the case of $\mathfrak{g}=\mathfrak{g} \mathfrak{u}(2)$ we have $([2])$

$$
\text { Pont }_{1}\left(g_{P}^{C}\right)=4 \text { Pont }_{1}(E)
$$

for the canonically induced complex vector bundle $E$ of rank 2 . This is derived in the following manner. Because $\operatorname{gr}(2, \boldsymbol{C})=\operatorname{gr}(2, \boldsymbol{C}) \oplus \boldsymbol{C}\left(\begin{array}{ll}1 & 0 \\ 0 & 1\end{array}\right)$, we have that $\mathrm{g}_{P}^{C} \oplus 1=E^{*} \otimes E$ for the dual $E^{*}$ of $E$. If we take the Chern character of both sides, then

$$
3+\frac{1}{2} \operatorname{Pont}_{1}\left(\mathrm{~g}_{P}^{C}\right)+1=4+c_{1}^{2}(E)[M]-4 c_{2}(E)[M],
$$

hence, $\operatorname{Pont}_{1}\left(\mathrm{~g}_{P}^{C}\right)=2\left(c_{1}^{2}(E)-4 c_{2}(E)\right)[M]=4 \operatorname{Pont}_{1}(E)-2 c_{1}^{2}(E)[M]$. Since the curvature form of an $S U(2)$-connection on $E$ is traceless, $c_{1}(E)=0$ from the ChernWeil theorem. Hence (4.4) is obtained.

\section{§5. Proof of Lemmas 3.2 and 3.3}

Lemmas 3.2 and 3.3, which were used to estimate $h^{2}=0$ in Section 3, are obtained by consequences of formula (3.10) in [5] which concerns with Bochner's type formula of the Laplacian of $d^{\nabla}$. In this section we deduce these lemmas by the aid of classical method in differential geometry.

Proof of Lemma 3.2. We show first that $d_{+}^{\nabla *}(\Omega \otimes \Phi)$ has the following expression;

$$
d_{+}^{\nabla *}(\Omega \otimes \Phi)=2 \sqrt{-1}\left(\partial^{\nabla} \Phi-\bar{\partial}^{\nabla} \Phi\right) .
$$

This is derived from the following consideration. By its definition $d_{+}^{\nabla^{*}}$ satisfies that $\left\langle d_{+}^{\nabla *}(\Omega \otimes \Phi), \Psi\right\rangle_{M}=\left\langle\Omega \otimes \Phi, d_{+}^{\nabla} \Psi\right\rangle_{M}$ for any $\mathrm{g}_{P^{\text {-valued }}}$ 1-form $\Psi=$ 
$\Psi^{+}+\overline{\Psi^{+}}\left(\Psi^{+} \in A^{1,0}\left(\mathrm{~g}_{P}^{C}\right)\right.$. Since $(1,1)$-part of $\quad d_{+}^{\nabla} \Psi$ is $\Omega \otimes\left\langle\bar{\partial}^{\nabla} \Psi^{+}+\partial^{\nabla} \overline{\Psi^{+}}, \Omega\right\rangle=$ $\Omega \otimes\left\{-\sqrt{-1} \Sigma_{\mu, \nu} g^{\mu \bar{\nu}}\left(\nabla_{\mu} \bar{\Psi}_{\nu}-\nabla_{\bar{\nu}} \Psi_{\mu}\right)\right\}$, the global inner product is given by

$$
\left\langle\Omega \otimes \Phi, d_{+}^{\nabla} \Psi\right\rangle_{M}=-2 \sqrt{-1} \int_{M} \sum g^{\mu \bar{\nu}} \operatorname{Tr} \Phi \cdot\left(\nabla_{\mu} \bar{\Psi}_{\nu}-\nabla_{\bar{\nu}} \Psi_{\mu}\right) d v .
$$

If we use Stokes' lemma; $0=\int_{M} \sum_{\mu, \nu} D_{\bar{\mu}}\left\{g^{\nu \bar{\mu}} \operatorname{Tr} \Phi \cdot \Psi_{\nu}\right\} d v$, then we have that

$$
\int_{M} \operatorname{Tr} \Phi \cdot\left(\sum g^{\nu \bar{\mu}} D_{\bar{\mu}} \Psi_{\nu}\right) d v=-\int_{M} \operatorname{Tr}\left(\sum g^{\nu \bar{\mu}} D_{\bar{\mu}} \Phi \cdot \Psi_{\nu}\right) d v
$$

By using this formula together with $\operatorname{Tr} \Phi \cdot\left[\Psi_{\mu}, \overline{A_{\nu}^{\nabla+}}\right]=-\operatorname{Tr}\left[\Phi, \overline{A_{\nu}^{\nabla+}}\right] \cdot \Psi_{\mu}$, we obtain the following

$$
\left\langle\Omega \otimes \Phi, d_{+}^{\nabla} \Psi\right\rangle_{M}=\left\langle 2 \sqrt{-1}\left(\partial^{\nabla} \Phi-\bar{\partial}^{\nabla} \Phi\right), \Psi\right\rangle_{M},
$$

from which (5.1) follows. Then it follows from (5.1) that $d_{+}^{\nabla} d_{+}^{\nabla *}(\Omega \otimes \Phi)=$ $2 \sqrt{-1}\left\{\partial^{\nabla} \circ \partial^{\nabla} \Phi+\Omega \otimes\left\langle\bar{\partial}^{\nabla} \circ \partial^{\nabla} \Phi-\partial^{\nabla} \circ \bar{\partial}^{\nabla} \Phi, \Omega\right\rangle-\bar{\partial}^{\nabla} \circ \bar{\partial}^{\nabla} \Phi\right\}$. Now apply (2.4) to this. Then we have that

$$
\begin{aligned}
d_{+}^{\nabla} \cdot d_{+}^{\nabla *}(\Omega \otimes \Phi)= & 2 \sqrt{-1} \Omega \otimes\left\langle\left[\Phi \wedge R^{\nabla}\right], \Omega\right\rangle \\
& -4 \sqrt{-1} \Omega \otimes\left\langle\partial^{\nabla} \bar{\partial}^{\nabla} \Phi, \Omega\right\rangle
\end{aligned}
$$

for the curvature form $R^{\nabla}$. Since $\left\langle\left[\Phi \wedge R^{\nabla}\right], \Omega\right\rangle=\left[\Phi,\left\langle R^{\nabla}, \Omega\right\rangle\right]=0$, (5.5) is reduced to $-4 \sqrt{-1} \Omega \otimes\left\langle\partial^{\nabla} \circ \bar{\partial}^{\nabla} \Phi, \Omega\right\rangle$.

Proof of Lemma 3.3. The operations of $\partial^{\nabla}$ on $\Phi \in A^{1,0}\left(\mathrm{~g}_{P}^{C}\right)$ and $\Psi \in A^{2,0}\left(\mathrm{~g}_{P}^{C}\right)$ are given as follows;

and

$$
\begin{aligned}
& \partial^{\nabla} \Phi=\frac{1}{2} \Sigma_{\mu, \nu}\left(\partial^{\nabla} \Phi\right)_{\mu \nu} d z^{\mu} \wedge d z^{\nu}, \\
& \left(\partial^{\nabla} \Phi\right)_{\mu \nu}=\tilde{\nabla}_{\mu} \Phi_{\nu}-\tilde{\nabla}_{\nu} \Phi_{\mu}
\end{aligned}
$$

$$
\begin{aligned}
& \partial^{\nabla} \Psi=\frac{1}{3 !} \sum_{\mu, \nu, \sigma}\left(\partial^{\nabla} \Psi\right)_{\mu \nu \sigma} d z^{\mu} \wedge d z^{\nu} \wedge d z^{\sigma}, \\
& \left(\partial^{\nabla} \Psi\right)_{\mu \nu \sigma}=\text { cyclic summation of } \tilde{\nabla}_{\mu} \Psi_{\nu \sigma} .
\end{aligned}
$$

We have the following for the operations of $\partial^{\nabla *}$ by the aid of (5.6) and (5.7);

for $\Psi \in A^{2,0}\left(\mathrm{~g}_{P}^{C}\right)$ and

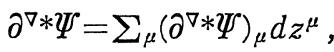

$$
\begin{aligned}
& \left(\partial^{\nabla * \Psi}\right)_{\mu}=-\sum_{\nu, \tau} g^{\nu \bar{\tau}} \tilde{\nabla}_{\bar{\tau}} \Psi_{\nu \mu}
\end{aligned}
$$

$$
\begin{aligned}
& \partial^{\nabla * \Theta}=\frac{1}{2} \Sigma_{\mu, \nu}\left(\partial^{\nabla * \Theta} \Theta\right)_{\mu \nu} d z^{\mu} \wedge d z^{\nu}, \\
& \left(\partial^{\nabla * \Theta}\right)_{\mu \nu}=-\Sigma_{\sigma, \tau} g^{\sigma \tau} \tilde{\nabla}_{\bar{\tau}} \Theta_{\sigma \mu \nu} .
\end{aligned}
$$

for $\Theta \in A^{3,0}\left(g_{P}^{C}\right)$.

Therefore we see that for $\Phi \in A^{2,0}\left(\mathrm{~g}_{P}^{C}\right)$ 
and

$$
\partial^{\nabla}\left(\partial^{\nabla * \Phi}\right)_{\mu \nu}=-\sum g^{\alpha \bar{\beta}}\left(\tilde{\nabla}_{\mu} \tilde{\nabla}_{\bar{\beta}} \Phi_{\alpha \nu}-\tilde{\nabla}_{\nu} \tilde{\nabla}_{\bar{\beta}} \Phi_{\alpha \mu}\right)
$$

$$
\partial^{\nabla *}\left(\partial^{\nabla} \Phi\right)_{\mu \nu}=-\sum g^{\alpha \bar{\beta}}\left(\tilde{\nabla}_{\bar{\beta}} \tilde{\nabla}_{\alpha} \Phi_{\mu \nu}+\tilde{\nabla}_{\bar{\beta}} \tilde{\nabla}_{\mu} \Phi_{\nu \alpha}+\tilde{\nabla}_{\bar{\beta}} \tilde{\nabla}_{\nu} \Phi_{\alpha \mu}\right),
$$

hence we have

$$
\begin{aligned}
& \left(\partial^{\nabla} \circ \partial^{\nabla *}+\partial^{\nabla *} \partial^{\nabla}\right) \Phi_{\mu \nu} \\
& =-\sum g^{\alpha \bar{\beta}} \tilde{\nabla}_{\bar{\beta}} \tilde{\nabla}_{\alpha} \Phi_{\mu \nu}-\sum g^{\alpha \bar{\beta}}\left(\left[\tilde{\nabla}_{\mu}, \tilde{\nabla}_{\bar{\beta}}\right] \Phi_{\alpha \nu}-\left[\tilde{\nabla}_{\nu}, \tilde{\nabla}_{\bar{\beta}}\right] \Phi_{\alpha \mu}\right) .
\end{aligned}
$$

By using the formula (1.19), we express the right hand side in terms of the curvatures of the Levi-Civita connection and the anti-self-dual $G$-connection $\nabla$. Then (5.10) is equal to

$$
\begin{aligned}
& -\sum g^{\alpha} \bar{\nabla}_{\bar{\nabla}} \tilde{\nabla}_{\alpha} \Phi_{\mu \nu}-\sum g^{\alpha \bar{\beta}}\left\{R^{\varepsilon}{ }_{\mu \bar{\beta} \alpha} \Phi_{\varepsilon \nu}+R_{\mu}{ }^{\bar{\beta}}{ }_{\nu} \Phi_{\alpha \varepsilon}\right. \\
& \left.+\left[\Phi_{\alpha \nu}, R_{\mu \bar{\beta}}^{\nabla}\right]-R_{\nu \bar{\beta} \alpha}^{\varepsilon} \Phi_{\varepsilon \mu}-R_{\nu \bar{\beta} \mu}^{\varepsilon} \Phi_{a \varepsilon}-\left[\Phi_{a \mu}, R_{\nu \bar{\beta}}^{\nabla}\right]\right\} \text {. }
\end{aligned}
$$

By the aid of the Rcici curvature tensor $R_{\bar{\alpha} \beta}$ of $g$ given by $R_{\bar{\alpha} \beta}=\sum g^{\mu \bar{\nu}} R_{\bar{\nu} \mu \bar{a} \beta}$ $=\sum g^{\mu \bar{\nu}} R_{\tilde{\alpha} \beta \bar{\nu} \mu}$, we can reduce the terms including the components of $R$ to $-\sum g^{\varepsilon \bar{\tau}}\left(R_{\bar{\tau} \mu} \Phi_{\varepsilon \nu}-R_{\bar{\tau} \nu} \Phi_{\varepsilon \mu}\right)$. Notice that the components $R_{\mu \bar{\beta} \alpha}^{\varepsilon}$ of $R$ are defined by $\left[D_{\mu}, D_{\bar{\beta}}\right] \xi_{\alpha}=\sum_{\varepsilon} R_{\mu \bar{\beta} \alpha}^{\varepsilon} \xi_{\varepsilon}$ (see [9]).

Now we assume finally that $M$ is complex two dimensional. Then, under the assumption that $g_{a \bar{\beta}}=\delta_{\alpha \beta}$ at a fixed point $x$, the above quantity is reduced to $-\left(R_{\overline{1} 1}+R_{\overline{2} 2}\right) \Phi_{\mu \nu}$. Since the scalar curvature $\rho$ is given by $\rho=-2 \sum g^{\alpha \bar{\beta}} R_{\bar{\beta} \alpha}$, this is equal to $(1 / 2) \rho \Phi_{\mu \nu}$.

The terms including $R^{\nabla}$ are also reduced to, under the same assumption that $g_{\alpha \bar{\beta}}=\delta_{\alpha \beta}$ at $x$,

$$
-\sum_{\alpha}\left(\left[\Phi_{a \nu}, R_{\mu \bar{a}}^{\nabla}\right]-\left[\Phi_{a \mu}, R_{\nu \bar{a}}^{\nabla}\right]\right) .
$$

Since it vanishes for $\mu=\nu$, it suffices to consider only the case of $\mu=1$ and $\nu=2$. It is reduced in this case to $-\left[\Phi_{12}, R_{1 \overline{1}}+R_{2 \overline{2}}\right]$, which vanishes from Proposition 2.2. Thus, the lemma is obtained by the above argument.

Note. The essential point that $M$ is Kähler is that the curvature tensor $R$ of $D$ is an $\operatorname{End}(T M)$-valued form of type $(1,1)$.

\section{References}

[1] Atiyah, M.F. and Bott, R., A Lefschetz fixed point formula for elliptic complexes, I, Ann. of Math., 86 (1967), 374-407.

[2] Atiyah, M.F., Hitchin, N.J. and Singer, I.M., Self-duality in four-dimensional Riemannian geometry, Proc. Royal. Soc. Lond. A, 362 (1978), 425-461.

[3] Atiyah, M.F. and Singer, I. M., The index of elliptic operators, III, Ann. of Math., 87 (1968), 546-604.

[4] Barth, W., Moduli of vector bundles on the projective plane, Invent. Math., 42 (1977), 63-91.

[5] Bourguignon, J.P. and Lawson, H. B., Stability and isolation phenomena for YangMills fields, Comm. Math. Phys., 79 (1981), 189-230. 
[6] Chen, B.-T., Some topological obstructions to Bochner-Kaehler metrics and their applications, Jour. Dif. Geom., 13 (1978), 547-558.

[7] Itoh, M., Moduli of anti-self-dual connections on Kähler manifolds, Proc. Japan Acad., 57A (1981), 176-180.

[8] Kobayashi, S. and Wu, H.H., On holomorphic sections of certain hermitian vector bundles, Math. Ann., 189 (1970), 1-4.

[9] Kodaira, K. and Morrow, J., Complex manifolds, 1971, Holt, Rinehalt \& Winston, Inc.

[10] Okonek, C., Schneider, M. and Spindler, H., Vector bundles on complex projective spaces, 1980, Birkhäuser.

[11] Tricerri, F. and Vanhecke, L., Curvature tensors on almost Hermitian manifolds, Transac. Amer. Math. Soc., 267 (1981), 365-398.

[12] Yang, C.N. and Mills, R.L., Conservation of isotopic spin and isotopic gauge invariance, Phys. Rev., 96 (1954), 191-195.

[13] Yau, S.-T., On the curvature of compact Hermitian manifolds, Invent. Math., 25 (1974), 213-239.

[14] Weil, A., Introduction a l'étude des variétés kählériennes, 1958, Hermann. 